\title{
Prediction of Motor Recovery Using Diffusion Tensor Tractography in Supratentorial Stroke Patients With Severe Motor Involvement
}

\author{
Kang Hee Kim, MD ${ }^{1}$, Yun-Hee Kim, MD, PhD ${ }^{1,2}$, Min Su Kim, MD $^{3}$, \\ Chang-hyun Park, $\mathrm{PhD}^{1}$, Ahee Lee, $\mathrm{MS}^{2}$, Won Hyuk Chang, MD, $\mathrm{PhD}^{1}$ \\ ${ }^{1}$ Department of Physical and Rehabilitation Medicine, Center for Prevention and Rehabilitation, \\ Heart Vascular and Stroke Institute, Samsung Medical Center, Sungkyunkwan University School of Medicine, Seoul; \\ ${ }^{2}$ Samsung Advanced Institute for Health Science and Technology, Sungkyunkwan University School of Medicine, Seoul; \\ ${ }^{3}$ Department of Rehabilitation Medicine, Wonkwang University School of Medicine, Iksan, Korea
}

Objective To investigate whether early stage diffusion tensor tractography (DTT) values predict motor function at 3 months after onset in supratentorial stroke patients with severe motor involvement.

Methods A retrospective study design was used to analyze medical records and neuroimaging data of 49 supratentorial stroke patients with severe motor involvement. Diffusion tensor imaging was assessed within 3 weeks after stroke in all patients. Three-dimensional tractography of the ipsilateral corticospinal tract (CST) was performed using the fiber assignment of the continuous tracking algorithm. The two-step DTT analysis was used. The first step was classification according to ipsilateral CST visualization. The second step was a quantitative analysis of the visible-CST group parameters. Motor function was assessed at 2 weeks and at 3 months after stroke. Comparative and correlation analyses were performed between DTT-derived measures and motor assessment scores.

Results Motor function of the upper extremity at 3 months after stroke was significantly higher in the visible-CST group than that in the nonvisible-CST group $(\mathrm{p}<0.05)$. Early stage fractional anisotropy was of DTT correlated significantly with upper extremity motor function at 3 months after stroke in the visible-CST group $(\mathrm{p}<0.05)$.

Conclusion These results demonstrate that early DTT-derived measures predict motor recovery in the upper extremity at 3 months after onset in supratentorial stroke patients with severe motor involvement.

Keywords Motor recovery, Corticospinal tract, Diffusion tensor tractography, Fractional anisotropy, FACT algorithm

Received February 5, 2015; Accepted April 3, 2015

Corresponding author: Won Hyuk Chang

Department of Physical and Rehabilitation Medicine, Center for Prevention and Rehabilitation, Heart Vascular and Stroke Institute, Samsung Medical Center, Sungkyunkwan University School of Medicine, 50 Irwon-dong, Gangnam-gu, Seoul 135-710, Korea

Tel: +82-2-3410-6068; Fax: +82-2-3410-0052, E-mail: wh.chang@samsung.com

(c) This is an open-access article distributed under the terms of the Creative Commons Attribution Non-Commercial License (http://creativecommons.org/ licenses/by-nc/4.0) which permits unrestricted noncommercial use, distribution, and reproduction in any medium, provided the original work is properly cited. Copyright $\odot 2015$ by Korean Academy of Rehabilitation Medicine 


\section{INTRODUCTION}

Motor impairment is a common complication after stroke and affects a patient's daily life [1]. Predicting motor recovery in patients with stroke is important for realistic goal setting and efficient resource allocation by clinicians and patients. A strong prognostic factor for motor recovery in patients with stroke is the degree of motor impairment at the acute phase [2]. However, inter-individual variability in initial impairment and subsequent motor recovery makes an accurate prognosis difficult [3].

Motor function in patients with stroke is strongly associated with the extent of injury and residual integrity of the corticospinal tract (CST) [4]. Understanding the precise CST status in patients with stroke is important to more accurately predict motor recovery. Diffusion tensor imaging (DTI) is an advanced magnetic resonance imaging (MRI) neuroimaging method that is useful for investigating motor tract integrity following stroke. Diffusion tensor tractography (DTT), which is derived from DTI, is used to three-dimensionally visualize neural tracts, which is not possible with conventional MRI [5]. DTI quantitative metrics obtained using a region of interest (ROI) analysis is limited to two dimensions. In addition, DTI does not provide information about the integrity of white matter fiber bundles. In contrast, DTT provides three-dimensional visualization of the architecture and integrity of specific white matter tracts [6]. Therefore, DTT has been proposed to predict motor recovery after stroke for verifying the integrity of the corticospinal tract.

Previous DTT studies [7-9] used semi-quantitative or qualitative analysis of the ipsilateral CST to predict motor recovery. However, these studies predicted motor function with DTT analysis at 3 to 4 weeks after stroke onset. Recent prospective studies on early rehabilitation after stroke have proven its safety, efficiency, and efficacy toward functional recovery $[10,11]$. Therefore, it is better to predict motor recovery after stroke as early as possible. In this study, we used DTT to analyze the relationship between visualization of the ipsilateral CST in the early stroke phase with motor recovery. In addition, a quantitative DTT analysis can improve the accuracy of previous semi-quantitative or qualitative analysis to predict motor recovery after stroke. Furthermore, we investigated if DTT values obtained at the early stroke stage predict motor function at 3 months after stroke.

\section{MATERIALS AND METHODS}

\section{Participants}

A retrospective study design was used to analyze the medical records of patients with stroke in our clinic who received a DTI scan within 1-3 weeks after stroke onset. Of 75 patients, 49 were included in the study according to the following criteria: 1) first-ever stroke, 2) cortical or subcortical unilateral stroke lesion confirmed by brain computed tomography (CT) scan or MRI, 3) severe motor function involvement (total Fugl-Meyer Assessment [FMA-T] motor score, 0-49) [12] on the day of the DTI scan, and 4) age 18-75 years. Exclusion criteria were any clinically significant or unstable medical disorders or any neuropsychiatric comorbidity other than stroke. This study was approved by the local ethics committee.

\section{DTI acquisition}

DTI data were collected using a 3 Tesla MR scanner. Forty-six whole brain images were acquired for every patient using a single-shot diffusion-weighted echo planar imaging sequence. The data set comprised 45 images with high diffusion weighting ( $b$ value $=1,000 \mathrm{~s} / \mathrm{mm}^{2}$ ) applied along 44 diffusion directions and one image with no diffusion weighting. Each image included $602.25-\mathrm{mm}$ thick axial slices of $1.96 \mathrm{~mm} \times 1.96 \mathrm{~mm}$ in-plane resolution.

\section{DTT processing}

CST fiber connectivity was evaluated using the fiber assignment by continuous tracking (FACT) algorithm and three-dimensional fiber reconstruction algorithm PRIDE software (Philips Medical Systems, Best, the Netherlands). Termination criteria for fiber tracking were fractional anisotropy (FA) $<0.2$ and angle change $>70^{\circ}[13,14]$. The two-ROI method was used for CST reconstruction, with ROIs including the motor cortex and lower anterior pons (Fig. 1). We excluded fibers connected to the cerebellum [15].

\section{Analysis of DTT}

A two-step DTT analysis was used. The first step was classifying the patients into two groups according to CST integrity in the affected hemisphere. The visible-CST group was comprised patients whose CST was preserved and the nonvisible-CST group was comprised of patients 

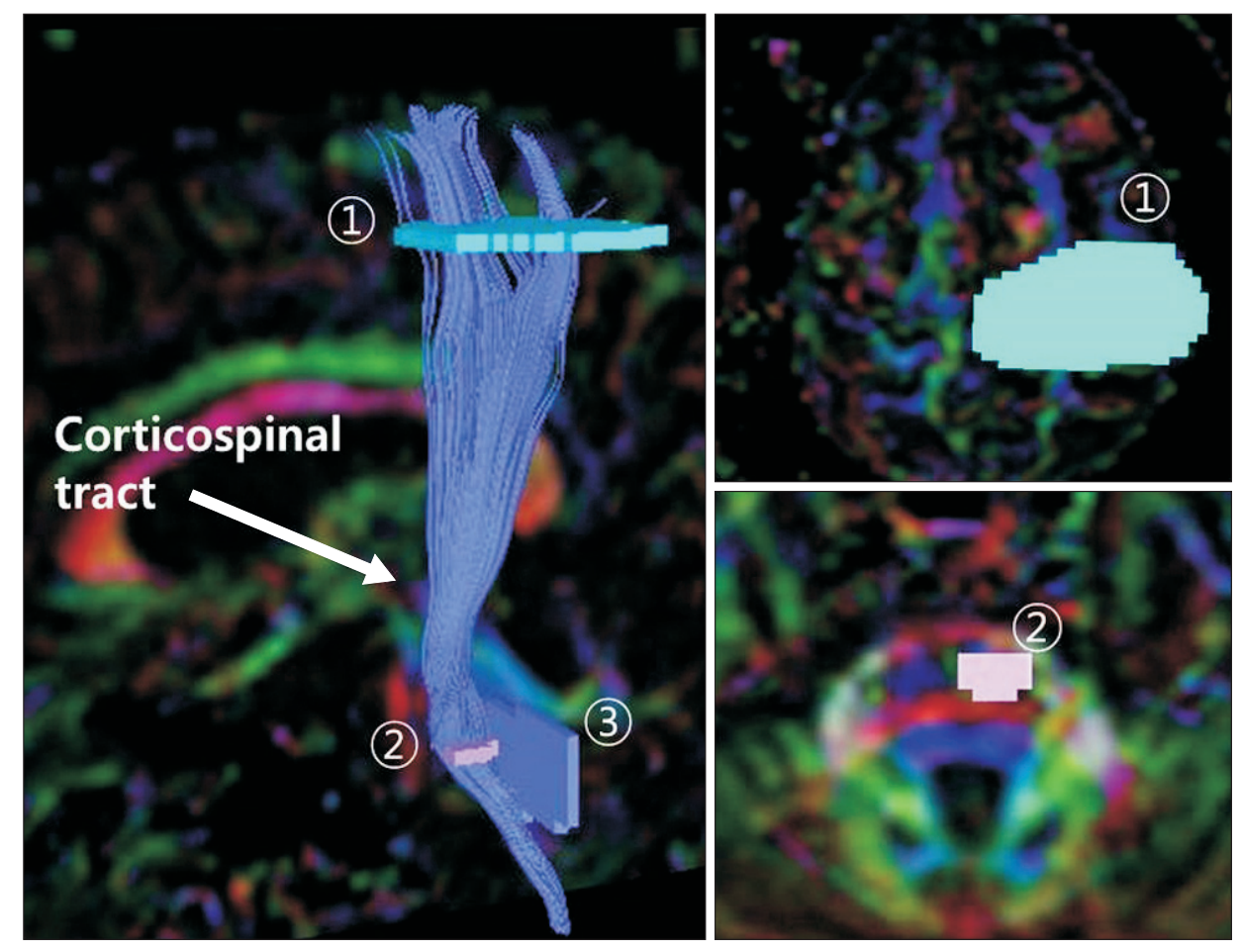

Fig. 1. Example of visualization of the corticospinal tract. (1), motor cortex; (2), lower anterior pons; (3), superior and middle cerebellar peduncle. whose CST was not preserved in the DTT analysis. The second step was a quantitative analysis of ipsilateral CST parameters in the visible-CST group by assessing FA and the number of fibers in affected and unaffected CSTs. The FA ratio and the number of fibers ratio were calculated as the affected value divided by the unaffected value [16].

\section{Motor function assessment}

Motor function of the affected extremity was measured in each patient on the same day with DTI and 3 months after stroke by FMA and the Motricity Index (MI) [17]. Motor function was assessed by a researcher who was blinded to DTT status.

The FMA motor score assessed for the upper and lower extremities. The upper limb motor score of the FMA (FMA-UL) is 0-66 and the lower limb motor score of FMA (FMA-LL) is 0-34. The maximum motor score for FMA-T was 100 . MI was used to measure motor function of the upper and lower extremities of the affected sides. Each score of the MI arm score (MI-A) and leg MI score (MI-L) is $0-100$. The MI side score of (MI-S) is the average of the MI-A and MI-L scores.

\section{Statistical analysis}

SPSS/PC+ software ver. 21.0 (SPSS Inc., Chicago, IL,
USA) was used for the statistical analysis. Independent t-tests and chi-square tests were used to compare the visible-CST and nonvisible-CST groups. The paired t-test was used to compare early phase values with values 3 months after stroke. A correlation analysis was used to assess correlation between motor function and quantitative DTT parameters for the affected CST in the visibleCST group. $p$-values $<0.05$ were considered statistically significant.

\section{RESULTS}

The general characteristics of the 49 patients are shown in Table 1. The visible-CST group had 30 patients, and the nonvisible-CST group had 19. The FMA-UL, FMA-LL, and FMA-T scores were significantly higher in the visible-CST group than those in the nonvisible-CST group at the early phase and 3 months after stroke onset $(\mathrm{p}<0.05)$. The MIA, MI-L, and MI-S scores were similar to the FMA scores. Motor function at 3 months after stroke improved significantly in both groups compared with those at the early phase as assessed by the FMA and MI $(\mathrm{p}<0.05)$ (Table 2).

Unaffected CST was preserved in DTT analysis in all patients. Table 3 shows the parameters in DTT of unaffected CST in all patients and of affected CST in the visible-CST 
Table 1. General characteristics of the patients

\begin{tabular}{lc}
\hline \multicolumn{1}{c}{ Parameter } & Value \\
\hline Age (yr) & $52.6 \pm 12.7$ \\
\hline Sex (male:female) & $24: 25$ \\
Type of stroke (ischemic:hemorrhagic) & $35: 14$ \\
Site of stroke (cortical:subcortical) & $23: 26$ \\
Side of stroke (right:left) & $20: 29$ \\
\hline Onset to DTI scanning (day) & $13.6 \pm 4.7$ \\
\hline
\end{tabular}

Values are presented as mean \pm standard deviation or number.

DTI, diffusion tensor imaging.

Table 2. Comparison between the visible and nonvisible corticospinal tracts

\begin{tabular}{lcc}
\hline & $\begin{array}{c}\text { Visible-CST group } \\
(\mathbf{n}=\mathbf{3 0})\end{array}$ & $\begin{array}{c}\text { Nonvisible-CST } \\
\text { group }(\mathbf{n}=\mathbf{1 9})\end{array}$ \\
\hline FMA-UL & & \\
Early phase & $9.9 \pm 6.5^{*}$ & $5.0 \pm 3.0$ \\
\hline 3 Months & $26.7 \pm 17.2^{* * * *}$ & $12.9 \pm 13.7^{* *}$ \\
FMA-LL & & \\
Early phase & $13.9 \pm 9.2^{*}$ & $8.6 \pm 7.4$ \\
\hline 3 Months & $23.5 \pm 7.6^{* * * *}$ & $16.6 \pm 8.3^{* *}$ \\
FMA-T & & \\
Early phase & $23.8 \pm 13.6^{*}$ & $13.6 \pm 9.3$ \\
\hline 3 Months & $50.2 \pm 22.4^{* * * *}$ & $29.5 \pm 19.5^{* *}$ \\
MI-A & & \\
\hline Early phase & $27.2 \pm 12.4^{*}$ & $11.4 \pm 12.6$ \\
\hline 3 Months & $50.0 \pm 20.9^{* * * *}$ & $27.4 \pm 21.7^{* *}$ \\
\hline MI-L & & \\
Early phase & $40.4 \pm 22.7^{*}$ & $28.9 \pm 20.9$ \\
\hline 3 Months & $61.4 \pm 20.3^{* * * *}$ & $43.1 \pm 24.0^{* *}$ \\
\hline MI-S & & \\
\hline Early phase & $34.4 \pm 17.0^{*}$ & $20.1 \pm 13.9$ \\
\hline 3 Months & $55.8 \pm 18.4^{* * * *}$ & $35.3 \pm 21.8^{* *}$ \\
\hline
\end{tabular}

Values are presented as mean \pm standard deviation.

CST, corticospinal tract; FMA, Fugl-Meyer Assessment; FMA-UL, upper limb motor score of FMA; FMA-LL, lower limb motor score of FMA; FMA-T, total score of FMA; MI, Motricity Index; MI-A, arm score of MI; MI-L, leg score of MI; MI-S, side score of MI.

${ }^{*} \mathrm{p}<0.05$ compared with the nonvisible-CST group.

${ }^{* *} \mathrm{p}<0.01$ compared with early phase in each group.

group. FA, the number of fibers, and their ratio were not correlated with upper motor function (FMA-UL and MIA) at the early phase of stroke in the visible-CST group.
Table 3. Diffusion tensor tractography parameters

\begin{tabular}{lcc}
\hline & $\begin{array}{c}\text { Affected CST in } \\
\text { visible-CST } \\
\text { group (n=30) }\end{array}$ & $\begin{array}{c}\text { Unaffected CST } \\
\text { in all patients } \\
(\mathbf{n}=\mathbf{4 9 )}\end{array}$ \\
\hline FA & $0.455 \pm 0.035$ & $0.517 \pm 0.028$ \\
FA ratio & $0.882 \pm 0.068$ & - \\
Number of fibers & $92.6 \pm 92.0$ & $213.2 \pm 84.4$ \\
\hline Number of fibers ratio & $0.470 \pm 0.617$ & - \\
\hline Vam
\end{tabular}

Values are presented as mean \pm standard deviation.

CST, corticospinal tract; FA, fractional anisotropy.

However, FMA-UL was significantly correlated with the FA value $(\mathrm{p}<0.05)$ and tended to be correlated with the FA ratio by DTT at the early stroke phase $(\mathrm{p}=0.051)$. In addition, MI-A at 3 months after stroke was significantly correlated with the FA value and the ratio by DTT at the early phase $(\mathrm{p}<0.05)$. No early phase DTT parameters correlated with FMA-LL, FMA-T, MI-L, or MI-S at the early phase or 3 months after stroke (Table 4 ).

\section{DISCUSSION}

In this study, patients with stroke and non-visualized ipsilateral CST by DTT at the early phase of stroke tended to have incomplete motor recovery at 3 months after onset. However, visualization of the ipsilateral CST by DTT at the early stroke phase correlated with moderate improvement in motor function at 3 months after stroke. In addition, motor recovery in the upper extremity was predicted by the DTT parameters during the early stroke phase. These results show that DTT at the early phase of stroke predicts motor recovery at 3 months after stroke. We suggest that a two-step DTT analysis according to ipsilateral CST visualization and a quantitative analysis of FA of visible ipsilateral CST is useful to predict motor recovery in early stroke patients with severe motor involvement.

DTT analysis is less susceptible to operator variability than DTI analysis and is more reproducible to localize tracts between patients $[18,19]$. Tractography algorithms can be classified into probabilistic and deterministic types [20]. Probabilistic algorithms generate tractography by creating multiple curves from seed points using Monte Carlo simulations. An example of a deterministic algorithm is the FACT algorithm. The FACT algorithm can easily change discrete voxel information in a continuous 
Table 4. Correlation analysis of motor function in the visible-CST group

\begin{tabular}{|c|c|c|c|c|c|c|c|c|c|c|c|c|}
\hline & \multicolumn{6}{|c|}{ Early phase } & \multicolumn{6}{|c|}{3 Months } \\
\hline & \multicolumn{3}{|c|}{ FMA } & \multicolumn{3}{|c|}{ MI } & \multicolumn{3}{|c|}{ FMA } & \multicolumn{3}{|c|}{ MI } \\
\hline & FMA-UL & FMA-LL & FMA-T & MI-A & MI-L & MI-S & FMA-UL & FMA-LL & FMA-T & MI-A & MI-L & MI-S \\
\hline \multicolumn{13}{|c|}{$x_{1}$} \\
\hline Value & $\begin{array}{c}0.287 \\
(0.124)\end{array}$ & $\begin{array}{c}0.098 \\
(0.606)\end{array}$ & $\begin{array}{c}0.203 \\
(0.283)\end{array}$ & $\begin{array}{c}0.113 \\
(0.551)\end{array}$ & $\begin{array}{c}0.064 \\
(0.738)\end{array}$ & $\begin{array}{c}0.018 \\
(0.927)\end{array}$ & $\begin{array}{c}0.401^{*} \\
(0.028)\end{array}$ & $\begin{array}{l}-0.006 \\
(0.976)\end{array}$ & $\begin{array}{c}0.305 \\
(0.101)\end{array}$ & $\begin{array}{r}0.420^{*} \\
(0.021)\end{array}$ & $\begin{array}{c}0.139 \\
(0.464)\end{array}$ & $\begin{array}{c}0.314 \\
(0.091)\end{array}$ \\
\hline Ratio & $\begin{array}{c}0.207 \\
(0.273)\end{array}$ & $\begin{array}{l}-0.034 \\
(0.857)\end{array}$ & $\begin{array}{c}0.075 \\
(0.694)\end{array}$ & $\begin{array}{c}0.105 \\
(0.581)\end{array}$ & $\begin{array}{c}0.038 \\
(0.841)\end{array}$ & $\begin{array}{c}0.006 \\
(0.976)\end{array}$ & $\begin{array}{c}0.359 \\
(0.051)\end{array}$ & $\begin{array}{l}-0.001 \\
(0.997)\end{array}$ & $\begin{array}{c}0.275 \\
(0.141)\end{array}$ & $\begin{array}{r}0.375^{*} \\
(0.041)\end{array}$ & $\begin{array}{c}0.115 \\
(0.545)\end{array}$ & $\begin{array}{r}0.276 \\
(0.140)\end{array}$ \\
\hline \multicolumn{13}{|c|}{ Number of fibers } \\
\hline Value & $\begin{array}{c}0.009 \\
(0.960)\end{array}$ & $\begin{array}{c}0.075 \\
(0.695)\end{array}$ & $\begin{array}{c}0.055 \\
(0.773)\end{array}$ & $\begin{array}{c}0.131 \\
(0.490)\end{array}$ & $\begin{array}{c}0.074 \\
(0.696)\end{array}$ & $\begin{array}{c}0.036 \\
(0.851)\end{array}$ & $\begin{array}{c}0.084 \\
(0.659)\end{array}$ & $\begin{array}{l}-0.004 \\
(0.983)\end{array}$ & $\begin{array}{c}0.063 \\
(0.741)\end{array}$ & $\begin{array}{c}0.148 \\
(0.436)\end{array}$ & $\begin{array}{c}0.040 \\
(0.833)\end{array}$ & $\begin{array}{r}0.106 \\
(0.577)\end{array}$ \\
\hline Ratio & $\begin{array}{l}-0.078 \\
(0.684)\end{array}$ & $\begin{array}{l}-0.014 \\
(0.941)\end{array}$ & $\begin{array}{l}-0.046 \\
(0.808)\end{array}$ & $\begin{array}{c}0.149 \\
(0.433)\end{array}$ & $\begin{array}{c}0.291 \\
(0.118)\end{array}$ & $\begin{array}{l}-0.042 \\
(0.827)\end{array}$ & $\begin{array}{l}-0.134 \\
(0.482)\end{array}$ & $\begin{array}{l}-0.127 \\
(0.504)\end{array}$ & $\begin{array}{l}-0.145 \\
(0.443)\end{array}$ & $\begin{array}{c}0.114 \\
(0.548)\end{array}$ & $\begin{array}{c}0.084 \\
(0.658)\end{array}$ & $\begin{array}{r}0.111 \\
(0.559\end{array}$ \\
\hline
\end{tabular}

Values are correlation coefficients (p-values).

CST, corticospinal tract; FMA, Fugl-Meyer Assessment; MI, Motricity Index; FMA-UL, upper limb motor score of FMA; FMA-LL, lower limb motor score of FMA; FMA-T, total score of FMA; MI-A, arm score of MI; MI-L, leg score of MI; MI-S, side score of MI; FA, fractional anisotropy.

${ }^{*} \mathrm{p}<0.05$.

track line. The line propagates in the vector direction of the pixels with discrete coordinates and can follow the actual tract more precisely. The FACT algorithm is easier to use for a DTT analysis than probabilistic algorithms $[21,22]$. Therefore, previous studies have used a DTT analysis with the FACT algorithm to predict motor recovery $[7,8,23,24]$. These studies reported that ipsilateral CST integrity determined by DTT during the early stroke stage predicts motor recovery in patients with a corona radiata infarction [7], pontine infarction [23], lacunar infarction [24], and intracerebral hemorrhage [8]. However, these studies only applied a semi-quantitative analysis with ipsilateral CST by DTT. FA of a nonvisible CST is calculated as zero in the quantitative analysis of DTT parameters, even though the threshold is set to $>0.2[25,26]$. This quantitative analysis has a limitation: CSTs with FA less than the threshold cannot be measured. Therefore, we used the two-step DTT analysis with a combination of previous qualitative and quantitative analyses.

We found that patients with visible ipsilateral CSTs had better motor recovery than patients with nonvisible ipsilateral CST by DTT during the early stroke phase. This result was consistent with previous DTT studies $[7,8,23,24,27]$. Parameters in patients with visible ipsilateral CST were significantly correlated with motor function of the upper extremity at 3 months after stroke but not during the early phase. These results suggest that motor recovery in patients with stroke is associated with residual ipsilateral CST integrity. They also suggest that residual ipsilateral CST integrity during the early phase of stroke is the basis for further motor function gains at 3 months after stroke. The lack of a significant correlation with motor function at the early phase may have been due to our inclusion criterion for severe motor involvement, which had a relatively short score range; however, our findings were consistent with a previous study [15]. We found no correlation between DTT parameters for the ipsilateral CST and motor function of the lower extremity. These results may have occurred because other motor fibers such as the corticobulbar and corticorubral tracts are also involved in motor function of the affected lower extremity in patients with stroke $[28,29]$. Predicting motor recovery of a lower extremity will require additional analytical methods for patients with stroke.

FA of DTT reflects injury of white matter fibers during pathological processes [30] and the number of fibers by DTT indicates the total volume of voxels with FA above a threshold for each depicted tract [15]. In our study, FA and the number of fibers by DTT did not significantly correlate with motor function in the upper extremity 3 months after stroke. However, a previous study in patients with stroke reported that the number of fibers by DTT significantly correlates with motor function at the chronic stage [15], although that study used a different 
protocol. Therefore, we cannot conclude whether the number of fibers by DTT has predictive value for motor recovery in patients with stroke.

This study had some limitations. We could not classify stroke in more detail. We included patients with hemorrhagic or ischemic stroke and with cortical or subcortical lesions. Stroke type and lesion were not homogenous in this study. For more accurate predictions of motor recovery, further studies with a larger number of patients are needed. DTT analysis with probabilistic algorithms appears to improve the identification of white matter tracts in lower FA regions, such as the cortex [13]. Although the FACT algorithm is easier to use, probabilistic algorithms could yield more accurate results, considering that the motor cortex was one of the ROIs in this study. This was one of the limitations. Despite these limitations, our results show the importance of ipsilateral CST integrity for goal-setting for stroke rehabilitation. The two-step DTT analysis that we used might predict motor recovery of the upper extremity in early supratentorial stroke patients with severe motor involvement.

\section{CONFLICT OF INTEREST}

No potential conflict of interest relevant to this article was reported.

\section{ACKNOWLEDGMENTS}

This study was supported by the Basic Science Research Program through the National Research Foundation of Korea (NRF) funded by the Ministry of Education, Science and Technology (20110014021) and the National Research Foundation of Korea (NRF) grant funded by the Korea government (MSIP) (NRF-2014R1A2A1A01005128).

\section{REFERENCES}

1. Schiemanck SK, Kwakkel G, Post MW, Kappelle LJ, Prevo AJ. Predicting long-term independency in activities of daily living after middle cerebral artery stroke: does information from MRI have added predictive value compared with clinical information? Stroke 2006;37:1050-4.

2. Stinear C. Prediction of recovery of motor function after stroke. Lancet Neurol 2010;9:1228-32.
3. Patel AT, Duncan PW, Lai SM, Studenski S. The relation between impairments and functional outcomes poststroke. Arch Phys Med Rehabil 2000;81:1357-63.

4. Langhorne P, Coupar F, Pollock A. Motor recovery after stroke: a systematic review. Lancet Neurol 2009; 8:741-54.

5. Melhem ER, Mori S, Mukundan G, Kraut MA, Pomper MG, van Zijl PC. Diffusion tensor MR imaging of the brain and white matter tractography. AJR Am J Roentgenol 2002;178:3-16.

6. Jang SH. The corticospinal tract from the viewpoint of brain rehabilitation. J Rehabil Med 2014;46:193-9.

7. Cho SH, Kim DG, Kim DS, Kim YH, Lee CH, Jang SH. Motor outcome according to the integrity of the corticospinal tract determined by diffusion tensor tractography in the early stage of corona radiata infarct. Neurosci Lett 2007;426:123-7.

8. Cho SH, Kim SH, Choi BY, Cho SH, Kang JH, Lee CH, et al. Motor outcome according to diffusion tensor tractography findings in the early stage of intracerebral hemorrhage. Neurosci Lett 2007;421:142-6.

9. Jang SH, Ahn SH, Sakong J, Byun WM, Choi BY, Chang $\mathrm{CH}$, et al. Comparison of TMS and DTT for predicting motor outcome in intracerebral hemorrhage. J Neurol Sci 2010;290:107-11.

10. Langhorne P, Stott D, Knight A, Bernhardt J, Barer D, Watkins C. Very early rehabilitation or intensive telemetry after stroke: a pilot randomised trial. Cerebrovasc Dis 2010;29:352-60.

11. Tay-Teo K, Moodie M, Bernhardt J, Thrift AG, Collier J, Donnan G, et al. Economic evaluation alongside a phase II, multi-centre, randomised controlled trial of very early rehabilitation after stroke (AVERT). Cerebrovasc Dis 2008;26:475-81.

12. Fugl-Meyer AR, Jaasko L, Leyman I, Olsson S, Steglind S. The post-stroke hemiplegic patient. 1: A method for evaluation of physical performance. Scand J Rehabil Med 1975;7:13-31.

13. Chang WH, Kim YB, Ohn SH, Park CH, Kim ST, Kim YH. Double decussated ipsilateral corticospinal tract in schizencephaly. Neuroreport 2009;20:1434-8.

14. Kunimatsu A, Aoki S, Masutani Y, Abe O, Hayashi N, Mori $\mathrm{H}$, et al. The optimal trackability threshold of fractional anisotropy for diffusion tensor tractography of the corticospinal tract. Magn Reson Med Sci 2004;3:11-7. 
15. Lindenberg R, Renga V, Zhu LL, Betzler F, Alsop D, Schlaug G. Structural integrity of corticospinal motor fibers predicts motor impairment in chronic stroke. Neurology 2010;74:280-7.

16. Yoshida S, Hayakawa K, Yamamoto A, Okano S, Kanda T, Yamori Y, et al. Quantitative diffusion tensor tractography of the motor and sensory tract in children with cerebral palsy. Dev Med Child Neurol 2010; 52:935-40.

17. Wade DT. Measuring arm impairment and disability after stroke. Int Disabil Stud 1989;11:89-92.

18. Murakami A, Morimoto M, Yamada K, Kizu O, Nishimura A, Nishimura T, et al. Fiber-tracking techniques can predict the degree of neurologic impairment for periventricular leukomalacia. Pediatrics 2008;122:500-6.

19. Rha DW, Chang WH, Kim J, Sim EG, Park ES. Comparing quantitative tractography metrics of motor and sensory pathways in children with periventricular leukomalacia and different levels of gross motor function. Neuroradiology 2012;54:615-21.

20. Yamada K, Sakai K, Akazawa K, Yuen S, Nishimura T. MR tractography: a review of its clinical applications. Magn Reson Med Sci 2009;8:165-74.

21. Li Z, Peck KK, Brennan NP, Jenabi M, Hsu M, Zhang $Z$, et al. Diffusion tensor tractography of the arcuate fasciculus in patients with brain tumors: Comparison between deterministic and probabilistic models. J Biomed Sci Eng 2013;6:192-200.

22. Mori S, van Zijl PC. Fiber tracking: principles and strategies: a technical review. NMR Biomed 2002;15: 468-80.

23. Jang SH, Bai D, Son SM, Lee J, Kim DS, Sakong J, et al.
Motor outcome prediction using diffusion tensor tractography in pontine infarct. Ann Neurol 2008;64:460-5.

24. Lai C, Zhang SZ, Liu HM, Zhou YB, Zhang YY, Zhang $\mathrm{QW}$, et al. White matter tractography by diffusion tensor imaging plays an important role in prognosis estimation of acute lacunar infarctions. Br J Radiol 2007;80:782-9.

25. Son SM, Kwak SY, Jang SH, Ahn SH, Cho YY, Kim HS, et al. Does injured corticospinal tract recover after rehabilitation therapy in patients with hemiplegic cerebral palsy? J Korean Acad Rehabil Med 2010;34:79-84.

26. Kwon JY, Chang WH, Chang HJ, Yi SH, Kim MY, Kim $\mathrm{EH}$, et al. Changes in diffusion tensor tractographic findings associated with constraint-induced movement therapy in young children with cerebral palsy. Clin Neurophysiol 2014;125:2397-403.

27. Kim EH, Lee J, Jang SH. Motor outcome prediction using diffusion tensor tractography of the corticospinal tract in large middle cerebral artery territory infarct. NeuroRehabilitation 2013;32:583-90.

28. Misawa S, Kuwabara S, Matsuda S, Honma K, Ono J, Hattori T. The ipsilateral cortico-spinal tract is activated after hemiparetic stroke. Eur J Neurol 2008;15:70611.

29. Ahn YH, Ahn SH, Kim H, Hong JH, Jang SH. Can stroke patients walk after complete lateral corticospinal tract injury of the affected hemisphere? Neuroreport 2006;17:987-90.

30. Lindenberg R, Zhu LL, Ruber T, Schlaug G. Predicting functional motor potential in chronic stroke patients using diffusion tensor imaging. Hum Brain Mapp 2012;33:1040-51. 